Zakharia K., « Genres poétiques et intra-textualité dans Sïrat al-Malik al-Zāhir Baybarș: L'exemple des trois premiers volumes » in Zakharia K. (coordonné par), Sïrat al-Malik al-Zāhir Baybarș/s, de l'oral à l'écrit/From Performance to Script, Arabica, tome LI, fascicules 1-2, Leyde, Brill, 2004, p. 189-211

\title{
Genres Poétiques et Intra-Textualité dans Sīrat Al-Malik al-Zāhir Baybarṣ: L'exemple des Trois Premiers Volumes
}

\author{
Par \\ Katia Zakharia \\ Université Lumière Lyon 2 *
}

Comme l'a signalé Georges Bohas dans sa contribution à ce numéro', nous avons pu, dans notre édition commune du texte arabe de Sīrat al-Malik al-Zāhir Baybarṣ (dans la recension de Damas), établir avec l'aide précieuse de l'informatique que cinquante-quatre citations poétiques, figurant dans les trois premiers volumes, se retrouvaient dans un ou plusieurs autres ouvrages.

Pour quarante-six de ces citations, il est légitime de parler d'emprunt. Elles sont en effet puisées dans un ouvrage relevant du patrimoine classique savant ; trente-trois ont pu être associées au nom du poète, célèbre ou moins connu, qui les a composées ${ }^{3}$. Lorsque les vers empruntés ne figurent que dans des sources tardives, ou y figurent aussi, on pourrait être tenté d'attribuer leur circulation entre les deux littératures au fait que les parois séparant 'amma et ḩāṣşa avaient commencé, aux alentours du XIV siècle, à perdre de leur étanchéité ; a fortiori plus tard. Pour autant, cet argument ne saurait servir à relativiser notre hypothèse selon laquelle le(s) "compositeur(s)" ${ }^{\prime 4} \mathrm{du}$ Roman aurai(en)t été cultivé(s), car une telle relativisation devient impossible quand on observe que certains vers ne figurent que dans une seule source connue et composée à une période bien antérieure. C'est le cas pour un vers mentionné à deux reprises ${ }^{5}$ et emprunté à la Yatīma ${ }^{6}$. On peut y associer un autre ${ }^{7}$ ne figurant que dans la Yatīma et le $\check{S}$ ír $r$ wa-al-šu 'arāer. L'absence de ces vers dans les sources classiques postérieures porte à proposer deux interprétations : la première consisterait à penser qu'ils auraient circulé oralement pendant des siècles sans retrouver de place dans le patrimoine écrit ; cela paraît

\footnotetext{
" Depuis 2015 CIHAM UMR 5648

${ }^{1}$ Voir supra p. 3-32.

${ }^{2} 2$ Sìrat al-Malik al-Zāhir Baybarș ḥasab al-riwāya al-šāmiyya ḥaqqaqa-hu wa- 'allaqa 'alay-hi Ğūrğ Būhās wa-Kātyā Zahariyya, Damas, Publications de l'Institut Français de Damas ; 2000, volume 1 ; 2001, volume 2 ; 2002, volume 3. ${ }^{3}$ Sur l'identité de ces poètes, voir infra l'article de G. Bohas.

${ }^{4}$ En l'état actuel de la recherche, je préfère cc terme à celui d'“auteur", quoiqu'il soit très tentant, au vu des particularités du texte et des éléments désormais connus de son histoire, de risquer l'hypothèse d'un auteur au sens habituel du terme. Mon “compositeur" est l'instance élaborant le texte, dans ce qu'elle a de mouvant, qu'il soit un ou plusieurs personnages.

${ }^{5}$ Baybars, 1, p. 173 et 3, p. 83 .

${ }^{6}$ Ouvrage du IX ${ }^{\circ}$ s. Son auteur, Ta'ālibī est mort en 1038.

${ }^{7}$ Baybars, 3, p. 337 .

${ }^{8}$ Ouvrage du IX ${ }^{\circ}$ s. Son auteur, Ibn Qutayba, est mort en 889.
} 
Zakharia K., « Genres poétiques et intra-textualité dans Sīrat al-Malik al-Zāhir Baybarș: L'exemple des trois premiers volumes » in Zakharia K. (coordonné par), Sīrat al-Malik al-Zāhir Baybarș/s, de l'oral à l'écrit/From Performance to Script, Arabica, tome LI, fascicules 1-2, Leyde, Brill, 2004, p. 189-211

peu probable, notamment quand on admet que les encyclopédies tardives, à l'instar du Mustațraf, ne se sont pas limitées à consigner les vers figurant dans les grands manuscrits savants.

La seconde interprétation, qui a ma préférence, consisterait à admettre que le "compositeur", familier des ouvrages tardifs, connaissait aussi des ouvrages plus anciens, au moins la Yatīma. Et, n'aurait-il connu que ce seul document, cela vaut d'être relevé et mérite de retenir l'attention.

En revanche, parler d'emprunts devient plus délicat pour les huit citations figurant à la fois dans le Roman et dans les Mille et une Nuits. Au sujet de ce dernier ouvrage, je reprends à mon compte la remarque de G. Bohas selon laquelle une partie de l'information nous échappe à l'heure actuelle, car nous ne disposons pas d'une version électronique des autres siyar ${ }^{\circ}$; autrement dit, qu'il reste à vérifier quels vers circulent entre l'ensemble des Romans pour parvenir à déterminer si ces ouvrages se les empruntaient les uns aux autres ou s'ils les puisaient également dans la mémoire collective, voire à une même source. À revoir aussi, ultérieurement, par comparaison avec le thesaurus d'un million de vers, disponible depuis peu, et encore inexistant quand nous avons entrepris ce travail ${ }^{\mathrm{n}}$, si d'autres citations ne trouvent pas leur pendant dans la littérature savante.

Ces deux dernières remarques n'empêchent pas de souligner déjà une interpénétration plus importante que beaucoup le pensaient jusqu'ici entre la littérature savante et cette littérature populaire, amenant à réviser un certain nombre d'affirmations considérées à ce jour comme définitives sur le patrimoine populaire en langue arabe. Dans cet article, je traiterai l'ensemble des citations poétiques figurant dans les trois premiers volumes du Roman, que leur auteur soit identifié ou non. Je commencerai par décrire rapidement l'ensemble du corpus. Je m'intéresserai ensuite à sa distribution par genres poétiques, puis aux marques textuelles annonçant ces citations et, enfin, aux personnages qui les déclament.

1. Nombre et distribution des citations poétiques

Les citations poétiques dont nous avons pu identifier l'origine ne constituent qu'une partie de celles contenues dans les trois premiers volumes de l'édition du texte arabe (les trente premiers des deux-

\footnotetext{
${ }^{9}$ Voir infra p. 29.

${ }^{10}$ On pourrait imaginer en faire une lecture linéaire exhaustive, un crayon à la main. Tout en admettant sans peine qu'il y a eu une vie avant l'informatique (et qu'il y en aura probablement plus d'une autre après), je préfère cependant confier au traitement automatique les aspects mécaniques de l'établissement de la documentation.

${ }^{11}$ Principalement, rappelons-le, à partir de la bibliothèque virtuelle www.alwaraq.com et du CD Rom al-Mawsū'a alši riyya, archivant un corpus de 500 ooo vers. Une nouvelle Mawsū'a ši riyya rassemblant plus d'un million de vers n'a pu être consultée pour ce travail.
} 
Zakharia K., « Genres poétiques et intra-textualité dans Sïrat al-Malik al-Zāhir Baybarș: L'exemple des trois premiers volumes » in Zakharia K. (coordonné par), Sīrat al-Malik al-Zāhir Baybarș/s, de l'oral à l'écrit/From Performance to Script, Arabica, tome LI, fascicules 1-2, Leyde, Brill, 2004, p. 189-211

cents fascicules du manuscrit) $)^{12}$. En effet, ces volumes incluent au total $135^{13}$ citations poétiques, représentant 631 vers. La source de 40\% de ces citations a donc pu être identifiée, en l'état actuel de la recherche. La répartition de la totalité de ces citations varie selon les fascicules et, partant, les volumes :

— 28 citations dans le premier volume (dont o8 identifiées) soit $28,6 \%$;

- 31 citations dans le second volume (dont og identifiées) soit $29 \%$;

— 76 citations dans le troisième volume (dont 35 identifiées) soit $46 \%$.

Si le nombre de citations s'accroît d'un volume à l'autre, cette progression ne peut être présentée comme une constante : le travail sur le prochain volume, sous presse à l'heure où j'écris ${ }^{14}$, permet déjà de le dire. Reste à déterminer, mais il faut pour cela que l'édition soit plus avancée, si ce nombre varie de manière sinusoïdale ou aléatoire.

2. Les "genres" poétiques

Principales caractéristiques du corpus en vers

Les thèmes abordés, les clichés, formules et topiques utilisés dans les vers, permettent de les classer et, de manière plus générale, de les répertorier selon un certain nombre de "genres" poétiques connus. Comme il n'est pas possible d'examiner dans le détail toutes ces citations dans le cadre du présent article, je commencerai par une rapide description de l'ensemble des composantes génériques de ce corpus et de leur distribution par genre ; puis, je montrerai, au moyen de trois exemples examinés de plus près (gazal, hamriyya, martiyya), comment les vers prennent sens, contextuellement, dans la dynamique interne du Roman. Les "genres" qui dominent les citations poétiques, que ce soit en nombre de citations ou en nombre de vers sont la poésie sapientielle ${ }^{15}$ et le faḩr (le plus souvent mêlé de tạhrị̄, en tous cas généralement lié aux batailles $\left.{ }^{16}\right)$.

\footnotetext{
${ }^{12}$ Rappelons que ces trois volumes représentent trente fascicules sur deux cents.

${ }^{13}$ Pour alléger la lecture, j'utiliserai les chiffres arabes pour tout ce qui concerne la description de la composition de ce corpus.

${ }^{14}$ Parution prévue fin 2003.

${ }^{15}$ J'entendrai ici par sapientiel, tout extrait vers exprimant une part de sagesse et/ ou de conseil, même s'il peut être secondairement rapporté à une autre typologie.

${ }^{16}$ Thème majeur de la Sīra, les batailles (dans leurs descriptifs, rites, rituels, ou formules littéraires) sont d'ailleurs examinées dans ce même numéro d'Arabica, dans leur dimension terrestre (J.-P. Guillaume) et maritime (A. R. Vidal Luengo); voir respectivement, p. 55-76 et 162-188.
} 
Zakharia K., « Genres poétiques et intra-textualité dans Sīrat al-Malik al-Zāhir Baybarș: L'exemple des trois premiers volumes » in Zakharia K. (coordonné par), Sīrat al-Malik al-Zāhir Baybarș/s, de l'oral à l'écrit/From Performance to Script, Arabica, tome LI, fascicules 1-2, Leyde, Brill, 2004, p. 189-211

Les citations à caractère sapientiel sont au nombre de $57\left(\right.$ soit $42 \%$ du corpus $\left.{ }^{17}\right)$. Elles traitent de ces thèmes convenus que les héros doivent pourtant avoir à l'esprit pour se débrouiller avec les réalités du monde. Elles rappellent ainsi que la roue tourne, que la Providence veille, que les bienfaits sont récompensés par les nobles et méconnus par les lâches, qu'il ne convient pas de divulguer les secrets, que les amis sont rares dans l'adversité, qu'il ne faut pas minimiser la jalousie meurtrière des adversaires et des méchants, que l'argent mène le monde... Prises isolément, quelques-unes de ces citations pourraient être rattachées à d'autres "genres" poétiques, comme ceux que l'on désigne par šakwāa al-dahr ou iḩwāniyyāt. J'ai considéré ici que si ces derniers se différenciaient par leur unité thématique et - éventuellement - par leur nombre de vers, on pouvait néanmoins les inscrire dans le cadre de la production poétique à portée sapientielle, si on en retient une définition élargie, d'autant que les citations examinées ici sont généralement courtes : totalisant 121 vers pour 57 citations, elles sont $79 \%$ à être composées seulement d'un ou de deux vers (27 dans le premier cas et 19 dans le second). Les personnages les plus divers les mentionnent pour appuyer les propos qu'ils adressent à leur entourage ou pour se conforter eux-mêmes dans leurs convictions.

Il faut noter que sept de ces citations sont récitées par le rāwī, qui intervient pour déclamer des vers à treize reprises (dans les trois volumes); ses six autres interventions relèvent du gazal (voir cidessous). Cette distribution permet de signaler déjà que le rāwī, qui n'a pas le monopole des thèmes poétiques dont il use lui-même, est exclu de certains types de poésie réservés aux héros intradiégétiques $^{18}$,tels le fah̆rou le madịh.

Les vers à caractère sapientiel

Dans les vers à caractère sapientiel, deux citations méritent d'être abordées un peu plus en détail. La première comprend seize vers ${ }^{19}$. Il s'agit d'un poème récité par le vizir Šāhīn devant ses collègues, pour les inciter à se réconcilier avec Baybarṣ contre lequel ils ont comploté et qui a décidé de ne plus avoir affaire à eux. Ces vers, qui font l'éloge de l'amitié, examinent son sens profond et décrivent les

\footnotetext{
${ }^{17}$ Baybarș, 1, p. 22, 24, 26, 28, 31, 33, 34, 36, 42, 44, 70, 144, 170, 178, 179, 19o, 252, 263, 309, 317 ; Baybarș, 2, p. 41, 48, 49, 125, 128, 137, 279, 335, 337 ; Baybars, 3, p. 27, 46, 59 (2) , 74, 75, 76, 102 (2), 104, 141, 174, 188, 189, 208, 225, 270, 279, 285-6, 286, $297,326,335,337,342,343,359$.

${ }^{18}$ Terme emprunté à G. Genette. Ces héros apparaissent à l'intérieur du récit rapporté par le rāwī qui y est lui-même extérieur. Cette configuration diffère de celle où, comme dans les Maqāmāt par exemple, le rāwīse retrouve parfois dans les deux positions.

${ }^{19}$ Baybarș, 3, p. 188.
} 
Zakharia K., « Genres poétiques et intra-textualité dans Sïrat al-Malik al-Zāhir Baybarș: L'exemple des trois premiers volumes » in Zakharia K. (coordonné par), Sīrat al-Malik al-Zāhir Baybarș/s, de l'oral à l'écrit/From Performance to Script, Arabica, tome LI, fascicules 1-2, Leyde, Brill, 2004, p. 189-211

comportements qui l'expriment, peuvent être rapportés sur le plan thématique, hors contexte, aux iḩwāniyyāt alors que leur fonction dans le récit relève du conseil. En réalité, c'est l'amitié qui lie Šāhīn et Baybarṣ qu'ils décrivent et non celle qui lierait ce dernier aux autres vizirs et chefs militaires, qu'ils paraissent décrire. Le vizir Šāhīn n'ignore pas que ses pairs n'ont pour Baybarṣ ni estime ni affection ; il feint de croire à leur sincérité parce qu'il entend les amener à présenter leurs excuses à celui sans lequel le pouvoir se transformera en chaos. Une autre citation de neuf vers ${ }^{20}$ peut être considérée comme le contre-point thématique de ce poème. Loin de peindre un mode de relations idéal fondé sur l'estime, le respect et l'affection, l'aubergiste Muhammad Manğak, auquel je reviendrai, y affirme crûment tout son mépris pour le rôle social de l'argent, qui inverse les valeurs, les certitudes et les vertus et parvient à dominer le monde : l'avidité de ses adversaires a précipité sa déchéance mais Baybarṣ interviendra pour le restaurer dans ses droits et sa dignité.

\section{Les vers de fahr}

25 citations (soit $18,5 \%$ du corpus) relèvent du fahr $r^{21}$. Elles totalisent 156 vers (soit près du quart du total). L'essentiel des propos qui y sont abordés a un caractère directement guerrier. Importantes sur le plan numérique, ces citations n'en sont pas moins réservées à des situations spécifiques : quatorze sont déclamées par des fidāwiyyeh ${ }^{22}$ et quatre par Baybarṣ au moment même où ils entrent sur le champ de bataille ${ }^{23}$, rappelant ainsi la tradition pré-islamiques d'associer jactance et invectives pour se donner du courage. En effet, si ces vers contiennent une part non négligeable d'attaques verbales contre l'ennemi, celui-ci, dans la plupart des cas, est trop éloigné pour les entendre. Ils visent donc moins la déstabilisation de l'adversaire que l'auto-émulation et/ ou l'encouragement des troupes. Si ces poèmes puisent leurs racines dans le passé pré-islamique, ils s'en démarquent systématiquement : le dernier vers est toujours associé à l'islam ${ }^{24}$, par le biais d'un éloge sacralisant le prophète Muhammad. Ce vers transforme rétrospectivement la signification de l'ensemble, valorisant le courage brutal ${ }^{25}$ revendiqué par le récitant en le mettant au service de la foi. Certes, la

\footnotetext{
${ }^{20}$ Baybarș, 3, p. 279.

${ }^{21}$ Baybarș, 1, p. 151 ; Baybarș, 2, p. 89, 113-14, 189, 211, 212, 296 ; Baybarș, 3, p. 71-2, 206, 209-10, 229, 230, 249, 256 , 257 (2), $257-8,258,324,35$ o (2), 351, 352, 352-3, 354-5.

${ }^{22}$ Les héros ismaïliens de la Sïra.

${ }^{23}$ Sur les rituels et formules propres à ces batailles, voir infra, la contribution de J.-P. Guillaume.

${ }^{24}$ Parfois, une autre allusion à Allah ou à l'islam traverse le poème.

${ }^{25}$ La plupart des images traitent, dans une sorte de "sereine violence", des effets du combat au corps à corps ou à l'arme blanche.
} 
Zakharia K., « Genres poétiques et intra-textualité dans Sīrat al-Malik al-Ẓahir Baybarș: L'exemple des trois premiers volumes » in Zakharia K. (coordonné par), Sïrat al-Malik al-Zähir Baybarș/s, de l'oral à l'écrit/From Performance to Script, Arabica, tome LI, fascicules 1-2, Leyde, Brill, 2004, p. 189-211

présence d'une telle poésie n’a rien de surprenant dans un roman guerrier; il n'en est pas moins intéressant de relever qu'elle est, le plus souvent, directement associée à des actions qui en authentifient le bien-fondé. Il ne s'agit pas de se proclamer courageux, mais de l'être et de le démontrer, en réalisant dans les faits les actes héroïques que l'on s'est attribué dans les vers (que ce soit par l'évocation de souvenirs ou par l'anticipation). Il ne s'agit pas davantage d'être courageux pour imposer sa force ou sa puissance, mais de l'être pour servir l'avènement d'un ordre islamique. Par ailleurs, en l'état actuel de notre recherche et malgré leur caractère fortement stéréotypé, seules quatre de ces citations incluent des segments ou des fragments pris chez d'autres poètes. Les emprunts au patrimoine classique sont ici plus de deux fois moins importants que pour l'ensemble du corpus. Ce n'est pas faute d'une représentation de ces thèmes dans ce patrimoine mais plutôt, et malgré la stéréotypie, en raison d'une nette personnalisation : les guerriers de la fiction rappellent leurs hauts faits et se glorifient de leur généalogie (notamment en référence à d'autres épisodes de la Sïra que ceux où les vers sont déclamés). En d'autres termes, c'est la nécessité narrative qui impose ici de manière patente et directe le contenu des passages versifiés. Le combattant trace par ses mots son auto-portrait idéalisé et finalisé au service de sa foi puis se lance dans le combat pour agir en conformité avec cette image.

Les vers de madịh

En ce qui concerne le madị $h^{26}$, son importance se mesure moins au nombre de citations (16) ou au nombre de vers (66) qu'à ce qui peut être désigné comme sa "spécialisation" ${ }^{{ }_{27}}$. En effet, malgré la profusion de personnages principaux ou secondaires susceptibles de faire l'objet d'un éloge ou d'un panégyrique dans la Sīra (notables, chefs militaires, divers vizirs ou princes, rois musulmans ou francs, etc.), cette poésie a deux héros seulement pour destinataires, à une exception près. Le premier destinataire du madịḥ est al-Ṣaliḥ Ayyūb. Jusqu'à sa mort (au début du troisième volume), c'est également le seul. Après son décès, on trouve le cas particulier de deux vers d'éloge récités par Baybarṣ pour accueillir le vizir Šāhīn qui lui rend visite, espérant le convaincre de renoncer à son deuil solitaire pour reprendre pied dans la vie de la cour. Par la suite, le madīh n'a plus que Baybar pour objet. Autre particularité : si les éloges servent parfois de prélude à une requête (demande de

\footnotetext{
${ }^{26}$ Baybarș, 1, p. 38, 193, 27 1, 284 ; Baybarș, 2, p. 129, 2 16, 257, 258, 298 ; Baybarș, 3, p. 61 (2), 19 1-2, 199, 259, 344, 355.

${ }^{27}$ Baybarș, 1, p. 38, 193, 27 1,274 ; Baybarș, 2, p. 129, 2 16, 257, 258,298 ; Baybarș, 3, p. 61 (2), 19 1-2, 199, 259, 344, 355.
} 
Zakharia K., « Genres poétiques et intra-textualité dans Sïrat al-Malik al-Zāhir Baybarș: L'exemple des trois premiers volumes » in Zakharia K. (coordonné par), Sīrat al-Malik al-Zāhir Baybarș/s, de l'oral à l'écrit/From Performance to Script, Arabica, tome LI, fascicules 1-2, Leyde, Brill, 2004, p. 189-211

soutien, de pardon...), ils n'ont jamais de lien avec une récompense d'ordre matériel. Les destinataires sont à l'évidence traités comme les titulaires du pouvoir mais à aucun moment comme de potentiels mécènes, pas même quand leur générosité est soulignée. Sur neuf fragments adressés au roi al-Ṣaăliḥ Ayyūb, c'est d'ailleurs Baybarṣ lui-même qui en récite cinq. Le lien tissé par le madịh entre les deux monarques conforte leur statut particulier dans le Roman. Ils sont, chacun à sa manière et selon son style, pareillement dignes de l'autorité qui leur est confiée et des compliments qu'on leur adresse.

Après cette présentation rapide des vers à caractère sapientiel, ceux relevant du madịḥ ou du fahrr, j'aborderai maintenant successivement et de manière un peu plus détaillée, les citations relevant de la poésie amoureuse, bachique ou élégiaque.

\section{Les vers de gazal}

Les vers d'amour, qu'ils soient de nasīb ou au gazal(gilmānīinclus), sont représentés par 21 citations (soit $15,5 \%$ du corpus), dont 12 ont un auteur identifié (soit $57 \%$ du corpus, c'est-à-dire largement au-dessus de la moyenne générale). Comme cela a été précisé plus haut, six de ces citations sont rapportées par le $r a \bar{w} \bar{T}^{\overline{8}}$, ce qui constitue près de la moitié de ses interventions, le reste étant consacré aux vers sapientiels. L'objet des vers d'amour, leur insertion dans le Roman ou leur détournement sont autant d'éléments qui informent le lecteur sur l'arrière-plan du récit. Ainsi, à trois reprises, ces vers servent à parler de l'amour d'une mère pour son enfant. À deux volumes d'intervalle, Sitt alŠām ${ }^{29}$ et Šağarat al-Durr ${ }^{30}$ citent le même vers, sensiblement dans les mêmes circonstances. La première le déclame en confiant son fils adoptif Baybarș au vizir Nağm al-Dīn al-Bunduqdārī (qui doit le conduire en Égypte), et la seconde en confiant son fils Halīl à Baybarṣ (qui veut le porter sur le trône). Dans les deux cas, la citation est précédée par le même acte : la mère prend la main de son fils et la met dans la main de celui auquel elle le confie. Enfin, dans ces deux exemples, le vers d'amour est déclamé en présence de celui qui en est l'objet, tout en étant adressé à un tiers. Ce sont également des vers de nasīb que récite la reine chrétienne convertie à l’islam, Maryam al-

\footnotetext{
${ }^{28}$ Baybarș, 1, p. 199 ; 2, p. 248 ; Baybarș, 2, p. 249-5o ; 3, p. 36 ; Baybarș, 3, p. 47 ; 3, p. 177.

${ }^{29}$ Baybars, 1, p. 173. Grande dame damascaine inspirée par une demi-soeur de Saladin, elle joue un rôle fondamental dans la formation de Baybarș.

${ }^{30}$ Baybars, 3, p. 83. Inspirée par la reine historique homonyme.
} 
Zakharia K., « Genres poétiques et intra-textualité dans Sïrat al-Malik al-Zāhir Baybarș: L'exemple des trois premiers volumes » in Zakharia K. (coordonné par), Sïrat al-Malik al-Zähir Baybarș/s, de l'oral à l'écrit/From Performance to Script, Arabica, tome LI, fascicules 1-2, Leyde, Brill, 2004, p. 189-211

Zunnāriyya ${ }^{31}$, après avoir été contrainte à abandonner son nouveau-né sur une île déserte, comptant sur la providence pour le sauver de la mise à mort certaine qui l'attend si elle le garde à ses côtés. Ces occurrences étant les seules dans lesquelles des vers d'amour sont récités par des femmes, il est possible de dire qu'utilisé par un personnage féminin dans notre corpus, le gazal exprime l'amour maternel. De manière plus générale, le Roman, s'il évoque régulièrement, le plus souvent de manière allusive, ce qui peut se passer entre un homme et une femme, est assez silencieux sur la façon dont cela est vécu par les femmes et à peine plus bavard pour ce qui est des hommes ${ }^{32}$. Une seule exception, mais elle est en prose : la description des émois (aussi physiques que naïfs ${ }^{33}$ ) de Maryam al-Zunnāriyya ${ }^{34}$ en présence de Ma 'rūf, émois pour lesquels elle se fera rappeler à l'ordre à deux reprises, et par l'intéressé et par le prisonnier musulman dont elle a fait son confident ${ }^{35}$.

Deux autres poèmes ${ }^{36}$ détournent les vers d'amour de leurs objets traditionnels. Il s'agit de ceux récités par le derviche derrière lequel se cache Šịhā Ğamāl al-Dīn, ce champion du déguisement et précieux allié de Baybarṣ. Dans les deux cas, il utilise le gazalpour exalter l'amour du divin. Mais, s'il parle de Dieu, son discours s'adresse directement à Baybarṣ auquel il a un message à transmettre. Il le fait une première fois en lui remettant le poème par écrit, la seconde en le déclamant. Mais il arrive aussi à Šịhā d'utiliser le gazal dans un cadre plus usuel. Déguisé en Bakrūma, jeune aubergiste chrétien, il agrémente une soirée en chantant un poème d'amour à la demande de son entourage ${ }^{37}$ qui le prend pour l'enfant prodigue enfin de retour. En effet, le véritable Bakrūma chantait fréquemment ces vers avant de quitter les siens. Le rāwī précise que Šịhā les avait mémorisés quelques instants plus tôt.

Inversement à l'utilisation par le derviche de vers parlant des amours humaines dans un contexte mystique, le récit détourne deux vers d'Ibn al-Fārị̣, relevant expressément du gazalmystique, pour parler des amours humaines ${ }^{38}$ : ils servent au rāwī à expliquer l'état de trouble dans lequel s'est

\footnotetext{
${ }^{31}$ Baybarș, 2, p. 277. Voir K. Zakharia, « A quoi rêvent les jeunes filles... ou les versions arabes de l'histoire de Maryam la Ceinturière », Langues et littératures du Monde Arabe (LLMA), 3, 2002.

${ }^{32}$ J'y vois, pour ce qui me concerne, moins un effet de censure qu'un effet de pudeur.

${ }^{33}$ Baybarș, 2, p. 247.

${ }^{34}$ Homonyme d'une héroïne des Nuits rappelons-le.

${ }^{35}$ Cela ne doit pas conduire à penser que les femmes ne jouent pas un rôle dans le Roman. Voir K. Zakharia, « Les principales figures de femmes musulmanes dans Sïrat Baybarș», LLMA, 4, 2003.

${ }^{36}$ Baybars, 3, p. 19 o et 26o.

${ }^{37}$ Baybars, 3, p. 377.

${ }^{38}$ Baybars, 2, p. 249.
} 
Zakharia K., « Genres poétiques et intra-textualité dans Sīrat al-Malik al-Zāhir Baybarș: L'exemple des trois premiers volumes » in Zakharia K. (coordonné par), Sīrat al-Malik al-Zāhir Baybarș/s, de l'oral à l'écrit/From Performance to Script, Arabica, tome LI, fascicules 1-2, Leyde, Brill, 2004, p. 189-211

trouvée la reine Maryam, quand le fidāwīMa'rūf s'est rendu chez elle à sa demande ${ }^{39}$. Rien ne permet de déterminer si ce détournement est fortuit ou délibéré. Le "compositeur" a pu choisir au hasard deux vers qu'il connaissait et qui lui paraissaient convenir dans ce contexte. Mais il a pu aussi bien décider de jouer sur une relative ambiguïté, dans la mesure où les amours de Maryam et de Ma 'rūf mêlent à la confusion des sentiments, les visions prémonitoires, la quête de la foi et la conversion à l'islam ${ }^{40}$.

Les citations restantes sont utilisées de manière plus habituelle. Deux concernent une fois de plus la reine Maryam. Une nuit, irrépressiblement attirée par son aimé, elle pénètre dans sa tente, défiant tous les usages et la bienséance. Elle tente de reproduire dans la réalité l'élan qui, dans un songe, l'avait jetée dans les bras de Ma 'rūf. Il se réveille et la regarde. Le rāwīintervient alors pour décrire en vers la beauté de Maryam et l'amour immense qu'elle pourrait inspirer ${ }^{41}$. Mais Ma'rūf y reste insensible, profondément choqué par la tache qui souillerait son honneur si la présence dans sa tente de cette femme, confiée à sa garde, venait à être connue. Il la congédie après l'avoir réprimandée.

Maryam arrive néanmoins à ses fins (conformes aux desseins divins) et le mariage est célébré. Peu de temps après, en l'absence de Ma 'rūf, la jeune femme est enlevée par les siens, fort mécontents et de sa conversion et de son union avec un musulman. Quand Ma 'rūf découvre la disparition de sa jeune épouse, à laquelle il s'est entre-temps beaucoup attaché et qui porte son enfant, il récite un poème de treize vers, utilisant les poncifs du nasī $b^{42}$. Rappelons que Maryam avait, quant à elle, utilisé le nasīb pour pleurer la séparation d'avec son nourrisson. Pour ce qui est de son enlèvement et de sa séparation d'avec Ma'rūf, elle récite pour sa part un poème triste ${ }^{43}$ dans lequel elle demande la miséricorde divine et un peu d'aide humaine, sans toutefois y exprimer de sentiments amoureux. Ibrāhīm, un autre fidāwī, jouant un rôle central dans le Roman $^{44}$, succombe aux charmes d'une belle, ismaïlienne comme lui, la labweh Nāfila al-Ḥaṣūn. Quand il la voit pour la première fois, elle est

\footnotetext{
${ }^{39}$ Baybarș, 2, p. 249-25o.

${ }^{40}$ Rappelons rapidement que Maryam avait vu en rêve Ma 'rūf, le chef fidāwīet décidé qu'il serait son époux. Pour aller à sa rencontre, elle prétexte un pèlerinage à Jérusalem, sachant qu'il devra alors assurer sa garde. Dans le même songe, elle voit un certain nombre de faits curieux, dont l'interprétation la conduira rapidement à se convertir à l'islam, devenu pour elle la seule vraie foi. Voir Zakharia K., « Maryam al-Zunnariyya... ».

${ }^{41}$ Baybarș, 2, p. 248.

${ }^{42}$ Baybars, 2, p. 273.

${ }^{43}$ Baybars, 2, p. 274.

${ }^{44}$ Voir infra, F. Guinle, « Ibrahim's embassys ».
} 
Zakharia K., « Genres poétiques et intra-textualité dans Sīrat al-Malik al-Zāhir Baybarș: L'exemple des trois premiers volumes » in Zakharia K. (coordonné par), Sīrat al-Malik al-Zāhir Baybarș/s, de l'oral à l'écrit/From Performance to Script, Arabica, tome LI, fascicules 1-2, Leyde, Brill, 2004, p. 189-211

habillée en garçon. Dans le récit rétrospectif qu'il fait à Baybarṣ de cette rencontre, et pour décrire son émotion à la vue de ce cavalier qui vient de mettre pied à terre près d'un point d'eau, il déclame deux vers en hommage à sa beauté45. Décontextualisés, ces deux vers pourraient être considérés comme relevant du ġazal gilmānī. Cependant, une prolepse ${ }^{46}$ subtile abolit une telle confusion. En effet, avant de réciter les vers, Ibrāhīm passe du masculin au féminin : « hayyāl muqbil... wa-huwa...

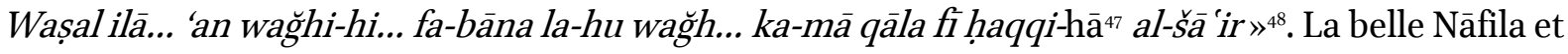

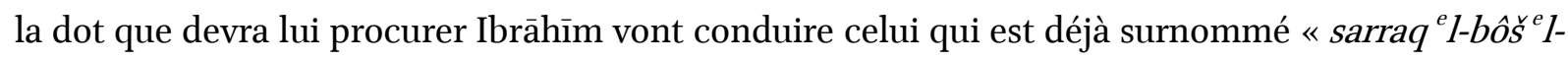
$m a h b \bar{u} l \gg^{49}$ à tenter de voler le bétail de sa propre famille.

La déraison qui accompagne la passion amoureuse atteint aussi le roi Aybak, adversaire de longue date du héros Baybarș. Il s'éprend d'une belle bédouine, proclame sa passion dans un mawwā $F^{\circ}$, l'épouse et s'installe avec elle, en oubliant ses fonctions royales et ses deux autres épouses. Il le paiera de sa vie : veuve d'al-Ṣāliḥ Ayyūb, la reine Šağarat al-Durr, qui a épousé Aybak en secondes noces, y voit un irréparable outrage... Mais avant qu'Aybak récite son mawwāl, le rāwïintervient pour décrire en vers la remarquable beauté de la jeune bédouine, comme pour justifier l'égarement du roi ${ }^{51}$. Un autre émir du roman est frappé par le mal d'amour et en subira d'amères désillusions. Il s'agit du bédouin, frère de lait de Baybarṣ, Muhammad. Envoyé par les siens à la recherche de Maḥmūd/Baybarṣ, qui avait été arraché à sa famille et vendu comme esclave, il s'éprend follement d'une almée et abandonne tout pour la suivre et la servir. Ayant retrouvé son frère, sans toutefois l'avoir reconnu, il lui raconte ce qui lui est advenu, incluant dans son récit trois fragments en vers. Les deux premiers, que Muhammad déclame pour rendre compte des origines de sa passion, sont

\footnotetext{
${ }^{45}$ Baybars, 3, p. 301.

${ }^{46}$ La prolepse temporelle est une anticipation (terme emprunté à G. Genette).

${ }^{47}$ C'est moi qui souligne.

${ }^{48}$ Baybars, 3, p. 301. Certains y verront peut-être une erreur de copiste. Une "erreur" qui serait si opportune s'impose comme procédé littéraire.

${ }^{49}$ Baybarș, 3, p. 30o. Les glissements de l'arabe dialectal à l'arabe littéral dans la Sīra, leurs modalités et leur interprétation ont été étudiés supra par D. E. Kouloughli. Comme cela a été souligné dans l'avertissement, les caractéristiques de la langue du Roman ont des incidences et sur la lecture et sur les transcription ou translittération. Faute d'une graphie conventionnelle unifiée pour restituer l'arabe syro-libanais en caractères latins, je m'accommoderai au mieux au texte :j'utiliserai, le o et le ô, pour rendre compte de la prononciation du $w a \bar{w}$ dans certains termes syro-libanais, le ${ }^{\mathrm{e}}$ pour la voyelle épenthétique produisant les liaisons ou remplaçant parfois certaines voyelles brèves. Je conserverai le qaf sous la forme q, qu'il puisse être produit q ou '. Enfin, si 'àmmiyya et fuṣhāa s'entremêlent dans un énoncé, j'utiliserai, pour la fușhāāle code de translittération d'Arabica et les italiques, et pour la ‘āmmiyya un code mélangé en caractères standard.

${ }^{50}$ Baybars, 3, p. 178.

${ }^{51}$ Baybars, 3, p. 177.
} 
Zakharia K., « Genres poétiques et intra-textualité dans Sīrat al-Malik al-Ẓahir Baybarș: L'exemple des trois premiers volumes » in Zakharia K. (coordonné par), Sīrat al-Malik al-Zāhir Baybarș/s, de l'oral à l'écrit/From Performance to Script, Arabica, tome LI, fascicules 1-2, Leyde, Brill, 2004, p. 189-211

communs au Roman et aux Nuits s. $^{2}$ L'un chante la beauté de l'almée, l'autre s'adresse à Allah, Lui rappelant que l'amour des hommes pour la beauté reflète Sa propre beauté et Son amour du Beau ${ }^{53}$. Mais, rapidement, la passion enthousiaste des premiers temps devient douloureuse puis insupportable ; Muḥammad décrit dans le dernier fragment, une souffrance, aussi inéluctable que l'amour qui la fonde, qui, affirme-t-il, le conduit progressivement dans la mort ${ }^{54}$.

Ce n'est pas seulement chez les personnages musulmans du Roman que les vers de gazal signalent les amours malheureuses. Dans le camp des chrétiens, c'est pour l'amour de la belle Rūmā que Sayf al-Rūm, le burțuquš, servant d'aide à l'ignoble Ğawān ${ }^{55}$, s'est engagé dans cette entreprise (qu'il n’apprécie que modérément). Rūmā est en effet la fille de son indigne mentor, qui lui a promis de lui accorder sa main s'il acceptait de le seconder loyalement ${ }^{56}$. Le rāwī explique, dans quatre vers empruntés au poète Bahā' al-Dīn Zuhayr ${ }^{57}$, comment la beauté de la jeune fille lui attache bien des cœurs, dont celui de Sayf al-Rūm. Quand le maître de ce dernier lui demande de réciter quelques vers d'amour, pour agrémenter une soirée, il répond aussitôt par une citation empruntée à Kutayyir, qui constitue une déclaration d'amour par anti-phrase («non, je ne confesserai pas mon amour pour 'Azza... » $)^{58}$. Enfin, c'est le diabolique Ğawān qui va, dans l'unique citation poétique qu'il déclame dans le corpus, vanter lui-même la beauté de sa fille ${ }^{59}$ pour reprendre de l'ascendant sur Sayf al-Rūm. Celui-ci, excédé par sa méchanceté, semblait prêt à se rebeller. Effectivement, les vers (pris ici chez Mağnūn) rendront au subalterne toute sa docilité. Il est intéressant de souligner que ces trois citations successives de gazal proviennent du patrimoine classique. À la fiction, rien d'impossible : Ğawān et Sayf al-Rūm parlent d'amour avec les mots des poètes 'udrītes, là même où le lecteur/auditeur n'aurait pas été surpris de voir les Francs du Roman déclamer pour la circonstance des vers de leur composition ; y compris dans un arabe approximatif, un peu à la manière de la reine Maryam pour laquelle Ma'rūf est son habỉbīet rūhịi. Mais, sans doute en raison du caractère universel attribué par la Sīra à la langue arabe et à son patrimoine, c'est de manière tout à fait banale que Ğawān et Sayf al-Rūm citent, pour évoquer l'amour, les vers les plus adaptés à de tels propos.

\footnotetext{
${ }^{52}$ Baybarș, 2, p. 82.

${ }^{53}$ Il s'agit la célèbre citation, inspirée par le ḥadīt : wa-anta ğamillun tuhibbu l-ğamāla fa-kayfa íbādu-ka lā ya šaqūna.

${ }^{54}$ Baybarș, 2, p. 84.

${ }^{55}$ Le double négatif de Baybarṣ.

${ }^{56}$ Ce qui n'empêchera pas Ğawān de faire à d'autres la même promesse. Voir, par exemple, Baybarș, 3, p. 326.

${ }^{57}$ Baybarș, 1, p. 199.

${ }^{58}$ Baybars, 3, p. 204.

${ }^{59}$ Baybarș, 3, p. 236-237.
} 
Zakharia K., « Genres poétiques et intra-textualité dans Sïrat al-Malik al-Zāhir Baybarș: L'exemple des trois premiers volumes » in Zakharia K. (coordonné par), Sīrat al-Malik al-Zāhir Baybarș/s, de l'oral à l'écrit/From Performance to Script, Arabica, tome LI, fascicules 1-2, Leyde, Brill, 2004, p. 189-211

Reste à évoquer trois citations de gazal gilmānī. Toutes trois figurent dans le même épisode, celui qui décrit l'accès au pouvoir, les comportements douteux et la mort accidentelle du roi 'Īsā al-Ġāzī, dont nous verrons aussi qu'il était particulièrement porté sur la boisson. Sollicité pour monter sur le trône, 'Īsā commence par refuser, craignant de devoir abandonner la vie de plaisir qui est la sienne. En effet, contrairement à son père, le très pieux roi al-Ṣaăliḥ, auquel Baybarṣ présume à tort qu'il

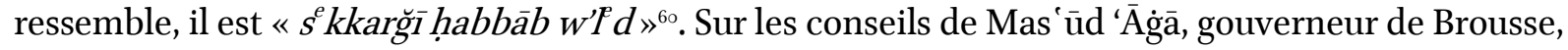
Rasūl 'Āg̀ā, l'émissaire de Baybarṣ, va demander au mamelouk Ğannantū/Ğannanto ${ }^{61}$ de l'aider à convaincre le prince héritier d'accepter le trône. Le rāwīintervient alors pour décrire la beauté de ce serviteur particulièrement influent auprès de son maître ${ }^{62}$. D'ailleurs, la manœuvre réussit.

Un peu plus tard, Baybarṣ étant venu à la rencontre du nouveau roi avec ses mamelouks d'élite, l'un d'entre eux, prénommé Bīleh, séduit — bien malgré lui — 'Īsā al-Ḡāzī par sa beauté. Le rāwī intervient une nouvelle fois pour décrire en vers les charmes de ce jeune homme ${ }^{6_{3}}$. À 'Īsā, qui s'enthousiasme avec insistance sur ses mamelouks, Baybarș en offre quelques-uns, qu'il lui laisse choisir, sans avoir décodé le véritable sens de l'intérêt que le roi leur manifestait. 'Īsā essaie vainement de soudoyer le jeune Bīleh pour le faire céder à ses désirs. Baybarș, témoin de la scène, à la demande même de son ancien serviteur, en conçoit alors un vif mépris pour le nouvel héritier du trône. Incapable de s'adapter aux comportements que l'on attend de lui, 'Īsā feint d'assurer ses fonctions mais s'arrange, avec la complicité de Ğannanto, pour avoir toujours à portée de main de quoi satisfaire son goût pour la boisson. Invité, selon l'usage, à suivre de loin le déroulement d'un combat, il demande à son serviteur de lui servir à boire et récite à cette occasion un petit poème ${ }^{64}$. L'objet de ce poème de gazal gilmānīpourrait sembler à première vue être Ğannantô lui-même. Une lecture plus attentive permet toutefois d'affirmer que c'est au contenu de son verre que le roi s'adresse. Si la poésie bachique traditionnelle nous a habitués, notamment chez Baššār Ibn Burd ou Abū Nuwās, à l'identification du vin à la femme aimée, l'équation vin= éphèbe est plus rare et peut être considérée comme l'une des (nombreuses) marques de littérarité et d'originalité du récit.

\footnotetext{
${ }^{60}$ Baybars, 3, p. 35.

${ }^{61}$ La graphie arabe est identique pour les deux. Lu avec ù, le prénom signifierait «vous l'avez rendu fou », avec o « je l'ai (ou tu l'as) rendu fou ».

${ }^{62}$ Baybars, 3, p. 36.

${ }^{63}$ Baybars, 3, p. 47.

${ }^{64}$ Baybars, 3, p. 69.
} 
Zakharia K., « Genres poétiques et intra-textualité dans Sīrat al-Malik al-Ẓahir Baybarș: L'exemple des trois premiers volumes » in Zakharia K. (coordonné par), Sīrat al-Malik al-Zāhir Baybarș/s, de l'oral à l'écrit/From Performance to Script, Arabica, tome LI, fascicules 1-2, Leyde, Brill, 2004, p. 189-211

Cet aperçu des citations de gazal permet d'esquisser une approche de leur fonctionnement contextuel général : les vers qui traitent de l'amour ne sont déclamés en présence de leur destinataire que lorsqu'ils détournent le discours sur l'amour, au sens convenu du terme, vers un autre discours ; c'est le cas de l'amour maternel ou encore, de l'amour divin dont témoigne Šịhā tout en s'adressant à Baybarṣ. Sinon, les vers de ġazal sont utilisés par le rāwīpour faire percevoir au lecteur/ auditeur la beauté d'un personnage, ou dans des récits à la première personne dans lesquels un héros tente de faire partager à son interlocuteur ce qui a motivé sa folie amoureuse (Ibrāhīm ou Maḥmūd, Sayf al-Rūm). Cela, qui n'est pas propre à ce seul Roman, n'en rend pas moins compte de la manière dont il traite spécifiquement cette poésie d'amour.

Trois situations se démarquent dans cet ensemble: le monologue récité par Ma rūf lors de l'enlèvement de Maryam, le ġazal ġilmānīutilisé par al-Ġāzī pour parler de sa passion pour la boisson et enfin, l'utilisation par Ğawān du gazalà des fins de manipulation.

\section{Les vers bachiques}

La hamriyya est représentée par deux citations seulement (soit 1,5\% du corpus) et 5 vers (moins de $1 \%$ du total). Ces citations figurent dans une même page ${ }^{6_{5}}$ et s'annulent réciproquement. La première est une libre restitution des quatre premiers vers du fameux poème d'Abū Nuwās contestant la conception du pardon divin chez le mu tazilite al-Naẓāām (da' 'anka lawm-ī...). La seconde inclut un seul vers, pris dans la Lāmiyya d'Ibn al-Wardī et préconisant une totale abstinence. Les deux fragments sont mentionnés par Muhammad Manğak. Dans une longue analepse, celui-ci raconte à Baybarṣ, qui se fait passer pour l'émir bédouin Fahīd, comment, ayant refusé de vendre le caravansérail dont il était propriétaire à Ġazza à un officier, ce dernier réussit à le circonvenir avec l'aide du qāậīdu lieu, en l'enivrant. Dans cet épisode, l'ivresse est présentée sans ambigüité comme une chose grave. Mais, plus grave encore que l'ivresse est l'injustice avec laquelle a été traité Muhammad Manğak : cette injustice est au centre de la séquence et son importance particulière est soulignée par l'intervention surnaturelle qui ouvre la narration : trois "revenants"66 expliquent à Baybarṣ qu'il devra faire halte là où le décidera son cheval pour sauver la victime d'une iniquité inacceptable. Cette victime n'est autre que Muhammad Manğak. Le lecteur/auditeur est donc

\footnotetext{
${ }^{65}$ Baybars, 3, p. 283.

${ }^{66}$ Baybarș, 3, p. 274-75
} 
Zakharia K., « Genres poétiques et intra-textualité dans Sīrat al-Malik al-Zāhir Baybarș: L'exemple des trois premiers volumes » in Zakharia K. (coordonné par), Sīrat al-Malik al-Zāhir Baybarș/s, de l'oral à l'écrit/From Performance to Script, Arabica, tome LI, fascicules 1-2, Leyde, Brill, 2004, p. 189-211

informé que ce personnage, de prime abord plutôt désagréable, et qui s’est laissé enivrer, n'en mérite pas moins d'être rétabli dans ses droits. Dans son récit, Manğak insiste sur le caractère intolérable de la transgression de l'interdit islamique de boire ${ }^{67}$. Très choqué de voir le qāộ̄is'y prêter et l'y inciter (par la première citation poétique), il refuse d'obtempérer (par la seconde), puis finit par s'exécuter sous la pression, de crainte d'être, sinon, mis à mort, alors qu'il a une famille à sa charge. Trois ans durant il expiera sa faute. Alors seulement, il découvrira qu'il avait, sous l'effet de l'ivresse, vendu son caravansérail. Ses adversaires avaient laissé passer le temps, non seulement pour réussir à le dépouiller totalement, mais aussi pour le faire travailler gratuitement à leur service. Il en vint à se demander si cette situation n'était pas le châtiment qu'il recevait pour sa ponctuelle, et bien involontaire, intempérance.

Baybarș interviendra pour appliquer la loi. Il sanctionnera ses propres représentants dans la région, ces dignitaires peu soucieux de l'ordre et de l'équité, ne s'informant pas dans le détail des affaires du royaume. Il punira également ceux qui ont mis au point cette "vente" illicite et transgressé, de surcroît, les interdits religieux. Enfin, il rendra à Manğak ses biens.

Le lecteur ne sera certes pas surpris de voir une place si réduite consacrée aux vers bachiques dans un Roman traitant de la restauration du pouvoir sunnite. La "mise en texte" de ce rejet n'en est pas moins intéressante, d'autant que dans les épisodes précédents, il avait été question de personnages buvant sans vergogne, notamment le roi 'Īsā al-Ġāzī, fils et successeur d'al-Șāliḥ Ayyūb ${ }^{68}$. La soif débordante d'al-Ġāzī, contrée par Baybarṣ, vaudra d'ailleurs au premier de mourir accidentellement peu après s'être repenti. Toutefois, al-Ġāzī ayant été présenté d'emblée par le narrateur comme un mauvais prince, ses comportements transgressifs en découlent logiquement et sa propension à trop boire sert à le tourner en ridicule ou à le montrer du doigt, dans les passages de prose, sans nécessiter d'être mis en exergue par la poésie.

\section{5. $\quad$ Les vers de ritāa}

Quoique la mort soit omniprésente dans le Roman, tant par les dangers constants que par les nombreuses exécutions, l'élégie funèbre n'apparaît qu'au début du troisième volume, représentée

\footnotetext{
${ }^{67}$ Baybars, 3, p. 282-284.

${ }^{68}$ Voir notamment Baybarș, 3, p. 49-52. On le sait, le Roman utilise aussi bien des faits que des figures historiques pour les adapter à sa cause. Dans l'étude des citations poétiques, je ne m'arrêterai donc pas aux interférences entre réalité et fiction.
} 
Zakharia K., « Genres poétiques et intra-textualité dans Sīrat al-Malik al-Ẓahir Baybarș: L'exemple des trois premiers volumes » in Zakharia K. (coordonné par), Sïrat al-Malik al-Zähir Baybarș/s, de l'oral à l'écrit/From Performance to Script, Arabica, tome LI, fascicules 1-2, Leyde, Brill, 2004, p. 189-211

seulement par 3 citations $(2,2 \% \text { du corpus })^{69}$ totalisant 5 o vers (près de $8 \%$ du corpus). Si le rităän'est pas beaucoup plus présent que la hamriyya pour ce qui est du nombre de citations, il est clair, vu le nombre de vers qui lui sont consacrés, que sa place dans la Sirra est plus importante. Ce nombre le rapproche davantage du madīh que de la poésie bachique. L'objet de la poésie funèbre représente un autre point commun avec le madịh: elle est exclusivement destinée au roi al-Ṣaliḥ (qui décède effectivement) et à Baybarș (supposé mort mais en fait bien vivant). La première de ces citations, la plus remarquable, est un poème de quarante-quatre vers. Pour deux de ces vers, le poète a été clairement identifié. Les autres sont truffés de clichés, formules, topiques ou thèmes si familiers, qu'ils laissent sur une impression de déjà connu. Il n'est pas à exclure que de plus amples vérification permettent d'attribuer d'autres vers à leur auteur, voire l'ensemble à un même poète. En effet, le poème est introduit par l'énoncé suivant : wa-li-llāhi darr man nazama hādihi al-marțiya al-adabiyya

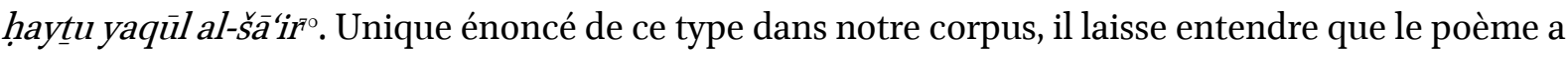
bien un auteur qui mérite les éloges, sans toutefois le nommer.

Cette élégie funèbre est récitée par al-Ṣālị̣ Ayyūb lui-même, anticipant sa propre mort, dans un récit qui entremêle le réalisme, les prédictions, l'intuition des fins dernières, les propos édifiants et la description de certains aspects de l'existence post mortem. Quelques instants avant de réciter ce poème, le roi blessé et se sachant mourant avait consolé Baybarṣ. Ce dernier, auquel il transmettait sa wașiyya, se tenait à ses côtés et appelait de tous ses vœux sa prompte guérison. Le roi lui avait rappelé la vanité du monde et le caractère inéluctable de la mort, puis avait récité ce poème, qui constitue la plus longue des citations poétiques des trois premiers volumes de la Sīra et, indubitablement, l'une des plus complexes sur le plan narratif, qu'il s'agisse d'un jeu sur l'espace, le temps et les voix, ou des mises en abîme successives... Il n'est pas possible d'entrer ici dans le détail de cette richesse littéraire. Il faut quand même souligner que ce poème pourrait faire à lui seul l'objet d'un long article.

Après une introduction générale de deux vers, rappelant que la mort est le suprême exil, le roi s'adresse à son âme lui rappelant qu'il n'est aucune provision, aucun bagage, aucun appui qui soit de quelque utilité pour ce voyage-là. Il va ensuite, dans les vers 5 à 10, insister sur la bonté divine opposée au caractère désobéissant et pécheur de l'homme qu'il est. Dans le vers 12, les larmes de

\footnotetext{
${ }^{69}$ Baybars, 3, p. 28-29, 31, 222.

${ }^{70}$ Baybars, 3, p. 28-29.
} 
Zakharia K., « Genres poétiques et intra-textualité dans Sïrat al-Malik al-Zāhir Baybarș: L'exemple des trois premiers volumes » in Zakharia K. (coordonné par), Sīrat al-Malik al-Zāhir Baybarș/s, de l'oral à l'écrit/From Performance to Script, Arabica, tome LI, fascicules 1-2, Leyde, Brill, 2004, p. 189-211

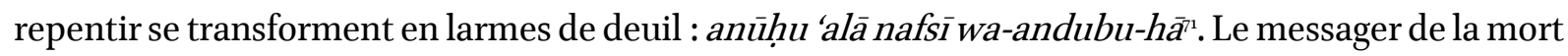
$(\text { rasūl al-mawt })^{7^{2}}$ attend de l'emporter. Il se voit malade, étendu sur sa couche parmi les siens, un médecin à son chevet. Au vers 16, la mort s'est emparée de son âme. Dédoublé, il voit son cadavre gisant ; il voit les siens, qui le pleurent, le recouvrir d'un linceul. Il les voit le laver. Il s'adresse alors à eux (souhait d'un agonisant ou voix d'outre-tombe ?), réclamant d'être lavé par un expert en la matière (vers 19). Il voit l'homme dévêtir son cadavre, l'étendre sur une planche, le laver avec célérité, le revêtir du linceul qui sera son dernier vêtement. Il voit les siens réciter les prières rituelles, l'accompagner jusqu'à sa tombe, l'y faire descendre, le pleurer, le recouvrir de terre puis le laisser. Seul dans la tombe, il continue de pleurer, effrayé par les questions que lui adressent Nakīr et Munkar. Dans le même temps, les siens se sont partagé son héritage, sa veuve s'est remariée et son enfant est maltraité par ceux qui l'entourent. Il se lamente encore devant ce que sont devenus son destin et le leur. À partir du vers $38^{73}$, il abandonne cette sorte de vision, revient au monde pour recommence à inciter son âme au repentir et à la vertu. Malgré la force qu'il dégage et le caractère fort peu réjouissant des thèmes qu'il aborde, le poème ne fait ensuite l'objet d'aucun commentaire, ni de la part des personnages ni de la part du rāwī. Le roi, sitôt le poème terminé, charge Baybarṣ d'une mission que ce dernier va accomplir. À son retour, il apprend qu'al-Ṣālih est mort.

Si al-Ṣāliḥ est particulièrement prolixe dans sa propre élégie funèbre, qu'il récite très peu de temps avant sa mort, Baybarṣ le pleurera seulement en deux vers ${ }^{74}$. Il exprimera sa douleur par d'autres voies, vivra son chagrin et son deuil en s'écartant longuement des sphères du pouvoir, avant d'être sollicité par les vizirs pour reprendre son activité. Il n'en demeure pas moins que ces deux vers, en eux-mêmes, par comparaison avec le poème présenté ci-dessus et qui les précède de peu, ou avec les cérémonies entourant la mort du roi, sont d'une banalité convenue et d'un laconisme partiellement décevants pour le lecteur/ auditeur. Ils ne sont pas personnalisés et n'évoquent même pas al-Ṣālih par son nom. Pourtant, si l'on en croit le rawī, Baybarṣ « [...] qu'Allah le prenne en Sa miséricorde - était le plus poète des gens de son temps, compétent en poésie, métrique et logique, connaisseur des langues arabes et de ce qui se trouve rassemblé dans un dictionnaire $»^{75}$. Deux

\footnotetext{
${ }^{71}$ Baybars, 3, p. 28.

${ }^{72}$ Id.

${ }^{73}$ Baybarș, 3, p. 29.

${ }^{74}$ Baybarș, 3, p. 31.

${ }^{75}$ Baybarș, 3, p. 261.
} 
Zakharia K., « Genres poétiques et intra-textualité dans Sīrat al-Malik al-Ẓahir Baybarș: L'exemple des trois premiers volumes » in Zakharia K. (coordonné par), Sīrat al-Malik al-Zāhir Baybarș/s, de l'oral à l'écrit/From Performance to Script, Arabica, tome LI, fascicules 1-2, Leyde, Brill, 2004, p. 189-211

lectures peuvent être faites de cette brièveté qui confine au mutisme : la première consiste à y voir un indice du sérieux et de la réserve du héros ; la seconde consiste à y voir seulement, ou y voir aussi, un indice du caractère singulier que revêtent dans la Sīra, et al-Ṣālị̣ et sa mort.

En effet, cette configuration dans laquelle c'est al-Ṣālih lui-même qui parle le plus longuement de son propre décès, confirme la fonction particulière du dernier sultan ayyūbide dans le roman : acteur de la fiction, il en est aussi l'une des figures symboliques les plus marquantes. Présent sur la scène du pouvoir, il vit à égale distance entre le Monde du Secret et le monde des vivants. Les autres le pleureront, certes ; mais, qui mieux que lui, qui a déjà un pied dans l'au-delà, aurait pu parler de cet ultime voyage, qu'il effectue, pour ce qui le concerne, en étant déjà au fait, par des moyens surnaturels, de ce qui adviendra après lui dans le monde terrestre de l'islam ?

La dernière citation relevant du ritāa ${ }^{76}$ est particulièrement touchante, puisque 'Uțmān pleure Baybars qu'il pense être mort assassiné et que le lecteur/auditeur sait, lui, être vivant. 'Uțmān, que l'on peut qualifier avec Jean-Patrick Guillaume de « saint rigolard » ${ }^{77}$ est véritablement désespéré. Il essaie de s'exhorter à la sagesse, à accepter le verdict divin, mais les propos sensés qu'il déclame, tout en témoignant de sa douleur, ne l'empêchent pas de donner sans retenue libre cours à celle-ci. Cette fausse mort qui joue, conjoncturellement, le rôle d'une ruse pour feinter l'ennemi et éprouver les alliés, a aussi, de manière plus générale, une fonction symbolique. Baybarș, qui était devenu du vivant du roi Aybak, le roi dissident al- Âdil, traverse à partir de cet événement qui a lieu durant la bataille d'al-'Arīš, une série d'épreuves (combats individuels, apparitions surnaturelles, circulation souterraine par des passages secrets, justice rendue là où on ne s'y attend guère, voyage sous un déguisement,...), avant de devenir ensuite le sultan al-Zāhir, successeur et alter ego de l'emblématique al-Ṣāliḥ. Et il n'est guère surprenant que ce soit 'Uțmān, son fidèle palefrenier, mais aussi son compagnon de route, auquel il incombe de le pleurer. 'Uțmān joue d'ailleurs, plus généralement dans le Roman, le rôle d'une mémoire. Non seulement il continue de temps en temps à appeler Baybarṣ « soldat Naybak $\gg^{78}$, son premier surnom à partir de son arrivée au Caire, mais il ne cesse d'évoquer auprès de divers personnages certaines de leurs actions qu'il a estimées répréhensibles. Ainsi, par exemple, le vizir Šāhīn n'est pas seulement pour lui le ḥăğğ Šăhūn $n^{79}$, mais

\footnotetext{
${ }^{76}$ Baybars, 3, p. 222.

${ }^{77}$ In Fleur de Truands, Sindbad, 1986 ; Actes Sud, 1998 ; p. 29.

${ }^{78}$ Écrit dans le texte al-kundī Naybak, et prononcé à l'égyptienne, pour ğundī. Voir par exemple, Baybarș, 3, p. 273.

79 'Uțman déforme régulièrement la plupart des noms et bon nombre de termes ou d'expressions.
} 
Zakharia K., « Genres poétiques et intra-textualité dans Sīrat al-Malik al-Ẓahir Baybarș: L'exemple des trois premiers volumes » in Zakharia K. (coordonné par), Sïrat al-Malik al-Zähir Baybarș/s, de l'oral à l'écrit/From Performance to Script, Arabica, tome LI, fascicules 1-2, Leyde, Brill, 2004, p. 189-211

surtout le wakkāl ${ }^{e}$ s-sukkar balāşso. Enfin, 'Uțmān, comme al-Ṣāliḥ, communique avec le Monde du Secret. Pourtant, rien n'est venu l'informer ici que la mort annoncée de son maître n'était qu'une ruse. Trop franc pour feindre, il n'aurait pu d'aucune manière simuler un chagrin qu'il n'aurait pas éprouvé. Il fallait donc qu'il soit trompé pour que les autres, plus retors, le soient aussi. Il est à noter également que le récit, s'il s'est étendu sur la douleur de 'Uțmān, persuadé d'avoir perdu un maître et un ami, est totalement silencieux sur la joie (ou la colère ?) que le pauvre palefrenier n'a pu manquer d'éprouver en apprenant que Baybarș était toujours vivant.

\section{Marqueurs de la citation poétique et représentation de la poésie}

En présentant rapidement les principales caractéristiques du corpus des vers, je pense avoir confirmé que l'emploi de ces citations ne relève pas d'une sorte de coutume littéraire ni d'une manière de réflexe conditionné consistant à en insérer ici ou là quelques-uns, mais bien d'un emploi organisé et faisant sens. Il est temps de dire maintenant quelques mots sur la manière dont la narration insère ces fragments dans le texte en prose, autrement dit la manière dont elle les annonce. Une étude complète de la fonction textuelle de ces annonces supposerait non seulement de décrire en détail la totalité du corpus mais aussi de vérifier si le choix d'un énoncé déterminé est lié à la nature des vers transmis ou à l'identité de leur transmetteur, ou peut-être aux deux. Cette analyse longue et très détaillée n'entre pas dans mon présent propos. Celui-ci sera limité à la présentation de quelques cas particulier puis, surtout, à esquisser la représentation qu'avaient de la poésie les “compositeur(s)" et récepteurs de la Sïra au vu de l'emploi qui en est fait.

Trois annonces de citations constituent des cas particuliers qui doivent être traités séparément. Les deux premières concernent des vers transmis dans une épître. Ils sont donc écrits et leur découverte se fait par la lecture, non par l'audition. Pour ces deux citations graphiées, le verbe introductif est d'ailleurs yarā: yarā fi-hi hāda al-bayt ${ }^{1}$, dans le premier cas, et yarā fi-hi maktūb hā dihi al-awzān lā ziyāda wa-la nuqșāă $7^{82}$ dans le second. Le premier fragment poétique écrit se trouve dans une missive, qui ne contient que ce seul vers, adressée par le roi al-Ṣaliḥ Ayyūb au fidāwī Ma rūf, le sommant d'assurer la protection de la princesse chrétienne Maryam al-Zunnāriyya. Le second fragment est un

\footnotetext{
${ }^{80}$ Baybarș, 3, p. 222 et passim. En référence à du sucre de canne que Baybarṣ lui avait offert à la grande fureur de 'Utman.

${ }^{81}$ Baybars, 2, p. 240.

${ }^{82}$ Baybars, 3, p. 261.
} 
Zakharia K., « Genres poétiques et intra-textualité dans Sīrat al-Malik al-Zāhir Baybarș: L'exemple des trois premiers volumes » in Zakharia K. (coordonné par), Sīrat al-Malik al-Zāhir Baybarș/s, de l'oral à l'écrit/From Performance to Script, Arabica, tome LI, fascicules 1-2, Leyde, Brill, 2004, p. 189-211

poème, consigné et remis par Šịhāa alors déguisé en derviche, à Baybarṣ. C'est un message codé dont le héros décryptera sans difficulté les significations. Le troisième et dernier cas particulier concerne une lacune du manuscrit : la citation annoncée à la fin du fascicule $27^{8_{3}}$ est absente au début du suivant $^{s_{4}}$. Elle est introduite par : s mi' wāḥad 'ammāl y qūl hādihi al-abyiāt $t^{s^{*}}$.

Quatre citations ${ }^{86}$ ne sont précédées d'aucune annonce et semblent s'insérer dans le texte comme la suite logique des propos qui les ont précédées. Elles figurent toutes dans le troisième volume, sont récitées par quatre personnages différents, dans des circonstances diversifiées, et traitent de thèmes variés. Je n'ai pu leur attribuer aucune fonction narrative ni même en suggérer une, sous forme d'hypothèse. À l'inverse, quatre citations sont précédées d'une annonce dans laquelle figurent l'indication de son caractère poétique et le nom du poète auquel les vers sont empruntés ${ }^{87}$. La première, élogieuse, concerne Baha' al-Dīn Zuhayr, présenté par sa kunya Abū al-Faḍl ; elle annonce de vers de ġazal qui lui sont empruntés. Dans ce savoureux mélange de fuṣhāà et de 'āmmiyya, qui fait l'un des attraits du texte, le rāwī annonce : m n g $^{\mathrm{e}}$ mlet mā wașaf-hā m ${ }^{\mathrm{e}} \mathrm{e}^{\mathrm{e}} \mathrm{s}$-šu" ār al-šà ír al-šahīr Abī (sic.) al-Fạ̣l al-amīr ḥaytu yaqūlu hādihi al-abyāt ${ }^{8}$. Cette formulation confirme que l'illustre poète tardif n'était pas inconnu de notre "compositeur".

Deux citations, toutes deux de fah̆r, sont annoncées comme un emprunt à 'Antara/'Antar. Le vers unique dont se compose la première est introduit par : fa-qultu la-hum mițl mā qāla 'Antar bnū 'Absī $(\text { sic. })^{89}$. Les deux vers de la seconde sont précédés de : wa-anā wa-llāhi la-a 'malu bi-mā qāla 'Antar $\operatorname{Banu}($ sic. $)$ 'Abs ${ }^{\circ}$. Le dernier poète nommé est Ibn al-Wardī dans le segment : a-mā sami'ta mā qāla Ibn al- $\operatorname{Ward}_{\overline{p^{1}}}$, introduisant le vers rappelant la prohibition du vin, mentionné plus haut.

Un énoncé signalant une prise de parole sans préciser qu'elle se fera en vers introduit 27 des extraits. Il s'agit d'énoncés comme wa-qāla, wa-qīla. Quelques marqueurs prennent directement à partie le destinataire des vers ; ceux-ci lui sont cités pour confirmer un conseil qui vient de lui être donné ou

\footnotetext{
${ }^{83}$ Baybars, 3, p. 277.

${ }^{84}$ Baybars, 3, p. 279.

${ }^{85}$ Baybars, 3, p. 277

${ }^{86}$ Baybarș, 3, p. 61, 141, 335, $35^{2}$.

${ }^{87}$ Baybarș, 1, p. 199 ; Baybarș, 2, p. 296 ; Baybarș, 3, p. 249, 283.

${ }^{88}$ Baybars, 1, p. 199.

${ }^{89}$ Baybars, 2, p. 296.

${ }^{90}$ Baybars, 3, p. 249.

${ }^{91}$ Baybars, 3, p. 283.
} 
Zakharia K., « Genres poétiques et intra-textualité dans Sīrat al-Malik al-Zāhir Baybarș: L'exemple des trois premiers volumes » in Zakharia K. (coordonné par), Sīrat al-Malik al-Zāhir Baybarș/s, de l'oral à l'écrit/From Performance to Script, Arabica, tome LI, fascicules 1-2, Leyde, Brill, 2004, p. 189-211

une suggestion qui vient de lui être faite. Le vers est pris comme un proverbe, un énoncé présentant une vérité générale.

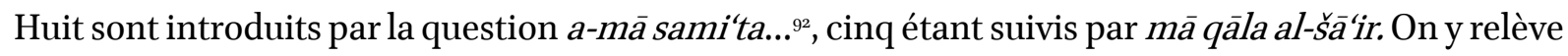
aussi un plaisant a-mā sami ta mā qālū hadôl ${ }^{93}$, illustrant cette forme particulière de littérarité dans laquelle l'enchevêtrement de la langue savante et de la langue parlée ne saurait être rapporté à une

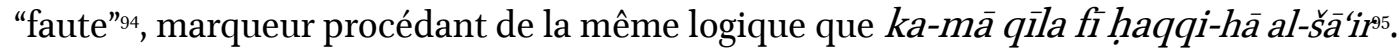

Mais l'un des aspects les plus intéressants des marqueurs de citation est qu'ils attestent que ni l'“amétricité", manifeste dans les exemples analysés par Georges Bohas ni le caractère aléatoire de la métrique dans les citations poétiques, même quand elles sont empruntées à d'illustres poètes, n'affectent leur perception dans la Sïra comme de la poésie, ayant un caractère de poéticité. Qu'il s'agisse de la nature du discours (vers), de la qualité de son auteur (poète) ou du comportement de son énonciateur (réciter, déclamer), aucune notion de correction ou d'erreur dans la composition métrique ne peut être relevée dans le texte ${ }^{96}$. En effet, pour ce qui est de la nature du propos, 29 marqueurs de citations incluent les termes bayt ou abyāt, auxquels on peut associer 2 occurrences

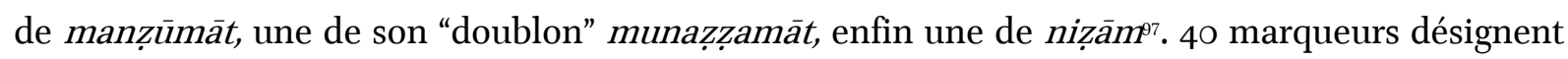
l'auteur de la citation par šā ît $^{98}$, s'ajoutant aux quatre occurrences dans lesquelles ledit poète est nommé. Quant au récitant, s'il advient que ses propos soient introduits par qāla ou ašādăa , ils le sont souvent par anšada $a^{\text {io }}$. Même si on exclut les énoncés dans lesquels deux de ces indications apparaissent en concomitance, comme wa-anšada yaqūlu hādayn al-baytayn ${ }^{10}$, il reste que le ilm al‘arū ne paraît pas conditionner ici ce qui est pris sans conteste pour de la poésie. Sans en être exclue, la métrique n'en est pas un constituant, ni pour le ḥakawātī ni pour son auditoire. Nous

\footnotetext{
${ }^{22}$ Baybarș, 1, p. 22, 33, 19 ; Baybarș, 2, p. 48, 49 ; Baybarș, 3, p. 102, 247, 270, 283, 297, 354-355.

${ }^{93}$ Baybarș, 2, p. 48.

${ }^{94}$ Voir note 49 .

${ }^{95}$ Baybars, 2, p. 248.

${ }^{96}$ À la différence de la prose, pour laquelle la Sīra a ses quelques critères de "normativité", nullement syntaxiques mais fondés sur l'appartenance ethnique (Francs, Turcs), régionale (Égypte, Syrie) ou plus rarement individuelle ('Uțān parle son propre égyptien).

${ }_{97}$ Baybarș, 1, p. 193, 199, 284 ; Baybarș, 2, p. 82, 84, 113-4, 129, 189, 240, 257, 258, 273, 277, 282 ; Baybarș, 3, p. 27, 31, 69, 71-2, 204, 209- 10, 229, 230, 238, 247, 257, 258, 261, 277, 279, 286, 287, 35०, 354-5, 377.

${ }^{9}$ Baybarș, 1, p. 22, 24, 26, 28, 33, 34, 36, 42, 44, 70, 170, 173, 178, 179, 19o, 199, 263, 317 ; Baybarș, 2, p. 49, 248, 249-50, 335, 337 ; Baybarș, 3, p. 28-9, 36, 46, 47, 59, 102, 177, 199, 225, 236-7, 261, 296, 301, 326, 342, 345, 347, 359 .

${ }_{99}$ Baybars, 1, p. 38 ; 2, p. 189, 258, 274, 277 ; 3, p. 350, 351.

${ }^{100}$ Baybarș, 1, p. 121, 193, 271, 274, 284; 2, p. 89, 113-114, 125, 211, 212, 216, 257, 273, 282, 298 ; 3, p. 61, 69, 76, 178, 189, 196, 199, 204, 206, 209-10, 222, 256, 257, 257-8, 259, 264-5, 283, 285-6, 287, 344, 351, 352-3, 355, 377.

${ }^{101}$ Baybarș, 1, p. 193.
} 
Zakharia K., « Genres poétiques et intra-textualité dans Sïrat al-Malik al-Zāhir Baybarș: L'exemple des trois premiers volumes » in Zakharia K. (coordonné par), Sīrat al-Malik al-Zāhir Baybarș/s, de l'oral à l'écrit/From Performance to Script, Arabica, tome LI, fascicules 1-2, Leyde, Brill, 2004, p. 189-211

sommes bien loin de l'allégorie du bédouin, qui, à l'instar d'un Monsieur Jourdain d'avant l'heure, faisait de la métrique sans le savoir, et tout aussi loin de l'impardonnable maksūr des savants ou poètes de métier.

\section{Les récitants}

Il reste à dire quelques mots de ceux qui, dans la Sïra, utilisent la poésie soit pour exprimer leurs sentiments soit pour inscrire leurs propos conjoncturels dans un cadre général, comme pour les référer à un modèle. Comme je l'ai indiqué plus haut, les personnages les plus variés citent des vers. Quelques-uns cependant se démarquent. La poésie est relativement fréquente dans les analepses, quand un personnage secondaire témoigne de son histoire ou qu'on la raconte. Ainsi, le marchand d'esclaves qui fera l'acquisition de Baybarș, 'Alī Āg̀a, en récite-t-il cinq face à ses déconvenues ; Muhammad Manğak, l'aubergiste enivré et trompé, en déclame sept ; Muḥammad, le frère de lait du héros, trois. Mais l'examen de l'identité des récitants permet surtout de voir dans la Sïra la principale démarcation narrative entre Baybarṣ et al-Ṣāliḥ, quand tout le reste les met en parallèle. En effet, le dernier des Ayyūbīdes ne déclame au total que quatre fragments en vers (à égalité avec 'Uțmān ou Šịha déguisé en derviche ${ }^{102}$ ). Par contre, avec trente-cinq citations, Baybarṣ non seulement le "dépasse" largement mais se pose comme le principal utilisateur de vers dans ces trois volumes, puisque le second est le rāwīavec treize citations seulement.

Mode d'expression digne d'un roi, la poésie apparaît aussi dans la Sïra, en opposition à une autre de ces images traditionnelles, comme le mode d'expression des vérités générales, des affirmations proverbiales et des descriptions précises. Le récit tranche quinze situations difficiles par șadaqa alšāir ou șadaqa man qāla suivis de vers. Et quelle meilleure conclusion donner à cet article que la réponse faite par Baybaq à son mentor et prédécesseur, qui vient de lui confier une importante mission :

Il a dit vrai, celui qui a dit :

Qui ne meurt par l'épée, mourra par autre chose ;

Diverses en sont les causes, mais il est une seule mort ${ }^{103}$.

Il ignorait que, sans cesse, la Sīra, le ferait renaître.

\footnotetext{
${ }^{102}$ Šịhā récite au total sept fragments.

${ }^{103}$ Baybarș, 2, p. 136-137.
} 